\title{
On analytical derivatives for geometry optimization in the polarizable continuum model
}

\author{
Helmut Harbrecht
}

Received: 1 May 2011 / Accepted: 9 June 2011 / Published online: 28 June 2011

(C) Springer Science+Business Media, LLC 2011

\begin{abstract}
The present paper is dedicated to the analytical computation of shape derivatives in the polarizable continuum model. We derive expressions for the interaction energy's sensitivity with respect to variations of the cavity's shape by means of the Hadamard representation of the shape gradient. In particular, by using the adjoint approach, the shape gradient depends only on two solutions of the underlying electrostatic problem. We further formulate boundary integral equations to compute the involved quantities.
\end{abstract}

Keywords Polarizable continuum model $\cdot$ Shape derivative $\cdot$ Boundary integral equations

\section{Introduction}

Continuum solvation models are widely used to model quantum effects of molecules in liquid solutions, see [17] for an overview. One of these models is known as the polarizable continuum model (PCM), introduced in [11]. In this model, the molecule under study (the solute) is located inside a cavity $\Omega$, surrounded by a homogeneous dielectric (the solvent). The solute-solvent interactions between the charge distributions which compose the solute and the dielectric are reduced to those of electrostatic origin.

The two central components of the model are the cavity construction and the solution of the electrostatic problem. The cavity is generally constructed as a set of interlocking spheres which leads to the van der Waals surface (VWS) or the solvent accessible surface (SAS). A more elaborate but also more accurate description employs the Connolly

H. Harbrecht $(\varangle)$

Mathematisches Institut, Universität Basel, Rheinsprung 21, 4051 Basel, Switzerland

e-mail: helmut.harbrecht@unibas.ch 
surface in order to faithfully represent the solvent excluded surface (SES) by "rolling" a sphere representing a solvent molecule over the cavity [5].

A convenient approach to resolve the electrostatic problem is provided by the reformulation as a boundary integral equation [1], also known as the integral equation formalism (IEF-PCM). In this way it is actually possible to apply the method to all cases where the Green's function for the considered environment is known. This has been done for liquid crystals (anisotropic permittivity) [4], ionic solutions (screened electrostatic potential) [1], sharp planar interfaces (image-charge approach) and diffuse planar interfaces (numerical integration) [8].

An important issue in molecular mechanics is the optimization of the molecule's geometry. It requires in particular the sensitivity of the interaction energy with respect to the change of the molecule's shape which is in general induced by the movement of the nuclei positions. Such a sensitivity analysis is the subject of shape optimization and meanwhile well established (see e.g. $[6,16]$ ). With the technique developed there, we derive in the present paper an exact and computable expression of the interaction energy's shape gradient. This is achieved by using the local shape derivative which is a measure of the sensitivity of the electric field with respect to the changes of the molecule's shape. By using additionally the so-called adjoint method, we derive the Hadamard representation of the shape gradient. It is given as an integral over the cavity's surface and involves the potentials and electric fields of the charges, i.e., only two transmission problems need to be solved in order to compute the complete shape gradient.

Let us emphasize that analytic expressions of the interaction energy's shape derivatives have firstly been derived many years ago in [2,3]. In comparison with these pioneering papers, our derivation of the shape gradient formula is much simpler. Moreover, we present integral equations to compute the ingredients. Nevertheless, the numerical application of our approach is postponed to a forthcoming paper.

Throughout the paper, we restrict ourselves to original PCM, i.e., we assume that the molecule is surrounded by a homogeneous dielectric. The molecule's surface can be arbitrarily shaped except for the assumption that it is $C^{1, \alpha}$-smooth with $\alpha \in(0,1)$ in order to ensure that the Hadamard representation of the shape gradient exists. However, this assumption can be weakened if only local shape perturbations are considered. Then, it suffices to provide $C^{1, \alpha}$-smoothness only at the support of these variations.

The paper is organized as follows. In Sect. 2 we formulate the specific transmission problem which arises from the electrostatic model under consideration. Section 3 is dedicated to the apparent surface charge and its computation. In Sect. 4 we compute the local shape derivative which is associated with the transmission problem's solution. It is used to derive the Hadamard representation of the exchange energy's shape gradient in Sect. 5. Finally, in Sect. 6, we present boundary integral equations which can be used to compute the ingredients of the shape gradient.

\section{Governing equation}

In quantum chemical simulations we are seeking the electronic wave function $\Psi$ : $\mathbb{R}^{3 N} \rightarrow \mathbb{R}(N$ denotes the number of electrons) such that the Schrödinger equation 
$\mathbb{E} \Psi=\mathcal{H} \Psi$ holds. In case of molecules in liquid solutions, the Hamilton operator splits into

$$
\mathcal{H}=\mathcal{H}_{\text {vacuum }}+\mathcal{H}_{\text {correction }}
$$

The correction term $\mathcal{H}_{\text {correction }}$ incorporates the solute-solvent interactions which are reduced to those of electrostatic origin.

We shall consider a cavity $\Omega$ with boundary $\Gamma:=\partial \Omega$ which represents the solutesolvent interface. The solvent in the exterior of $\Omega$ is represented by a constant dielectric medium. The solute and thus the support of the wave function $\Psi$ is assumed to be contained inside the cavity. Therefore, the correction term in (2.1) refers to the interaction energy between charges $\rho, \rho^{\prime}$, located inside the cavity $\Omega$, i.e., $\operatorname{supp}(\rho), \operatorname{supp}\left(\rho^{\prime}\right) \subset \bar{\Omega}$. It is given by

$$
E_{I}\left(\rho, \rho^{\prime}\right)=\int_{\mathbb{R}^{3}} u(\mathbf{x}) \rho^{\prime}(\mathbf{x}) \mathrm{d} \mathbf{x}
$$

with the electrostatic potential $u \in H_{l o c}^{1}\left(\mathbb{R}^{3}\right)$ satisfying the following transmission problem:

$$
\begin{array}{ll}
-\Delta u_{i}=\rho & \text { in } \Omega, \\
\Delta u_{e}=0 & \text { in } \Omega^{c}, \\
u_{i}=u_{e}, \frac{\partial u_{i}}{\partial \mathbf{n}}=\epsilon \frac{\partial u_{e}}{\partial \mathbf{n}} & \text { on } \Gamma, \\
\left|u_{e}(\mathbf{x})\right|=\mathcal{O}\left(\|\mathbf{x}\|^{-1}\right) & \text { as }\|\mathbf{x}\| \rightarrow \infty .
\end{array}
$$

Herein, $\mathbf{n}$ is the outward unit normal at $\Gamma$ and $\epsilon>0$ is the macroscopic dielectric constant of the solvent outside $\Omega$. The representation of the local shape derivative (see Sect. 4) requires $u \in H_{l o c}^{1}\left(\mathbb{R}^{3}\right) \cap\left(H^{2}(\Omega) \cup H_{l o c}^{2}\left(\Omega^{c}\right)\right)$ which is satisfied provided that $\Gamma$ is of class $C^{1, \alpha}$ with $\alpha \in(0,1)$, see [7].

\section{Apparent surface charge}

In order to compute the interaction energy (2.2), we shall introduce some boundary integral operators. Associated with the interior of the cavity $\Omega$, define the single and double layer operator of the Poisson equation

$$
(\mathcal{V} u)(\mathbf{x})=\int_{\Gamma} \frac{u(\mathbf{y})}{4 \pi\|\mathbf{x}-\mathbf{y}\|} \mathrm{d} o_{\mathbf{y}}, \quad(\mathcal{K} u)(\mathbf{x})=\int_{\Gamma} \frac{\langle\mathbf{n}(\mathbf{y}), \mathbf{x}-\mathbf{y}\rangle}{4 \pi\|\mathbf{x}-\mathbf{y}\|^{3}} u(\mathbf{y}) \mathrm{d} o_{\mathbf{y}}, \quad \mathbf{x} \in \Gamma
$$

and the related Newton potential

$$
\mathcal{N}_{\rho}(\mathbf{x})=\int_{\Omega} \frac{\rho(\mathbf{y})}{4 \pi\|\mathbf{x}-\mathbf{y}\|} d \mathbf{y}, \quad \mathbf{x} \in \mathbb{R}^{3}
$$


Following [1], the sought interaction energy (2.2) can be expressed in terms of an apparent surface charge $\sigma \in H^{-1 / 2}(\Gamma)$.

Theorem 1 The interaction energy between two charges $\rho, \rho^{\prime} \in \widetilde{H}^{-1}(\Omega)$ is given by

$$
E_{I}\left(\rho, \rho^{\prime}\right)=\underbrace{\int_{\mathbb{R}^{3} \mathbb{R}^{3}} \int_{\text {correction term: }\left(\sigma, N_{\rho^{\prime}}\right)_{L^{2}(\Gamma)}} \frac{\rho(\mathbf{x}) \rho^{\prime}(\mathbf{y})}{4 \pi\|\mathbf{x}-\mathbf{y}\|} \mathrm{d} \mathbf{x} \mathrm{d} \mathbf{y}}_{\text {exchange energy in the vacuum }}+\underbrace{\iint_{\mathbb{R}^{3}} \frac{\rho^{\prime}(\mathbf{x}) \sigma(\mathbf{y})}{4 \pi\|\mathbf{x}-\mathbf{y}\|} \mathrm{d} \mathbf{x} \mathrm{d} o_{\mathbf{y}}}_{\Gamma \mathbb{R}^{3}}
$$

where the apparent surface charge $\sigma=\mathcal{V}^{-1}\left(u_{i}-\mathcal{N}_{\rho}\right) \in H^{-1 / 2}(\Gamma)$ satisfies the boundary integral equation

$$
\mathcal{V} \sigma=\frac{1}{\epsilon-1} \mathcal{A}^{-1} \mathcal{N}_{\rho}-\mathcal{N}_{\rho}, \quad \mathcal{A}:=\frac{\epsilon+1}{2(\epsilon-1)}-\mathcal{K}, \quad \text { on } \Gamma .
$$

Proof There hold the Dirichlet-to-Neumann maps [14]

$$
\mathcal{V} \frac{\partial u_{i}}{\partial \mathbf{n}}=\left(\frac{1}{2}+\mathcal{K}\right) u_{i}-\mathcal{N}_{\rho}, \quad \mathcal{V} \frac{\partial u_{e}}{\partial \mathbf{n}}=\left(\mathcal{K}-\frac{1}{2}\right) u_{e} \text { on } \Gamma
$$

Thus, in view of the jump conditions (2.3), resolving for $\sigma:=\mathcal{V}^{-1}\left(u_{i}-\mathcal{N}_{\rho}\right)$ yields immediately (3.3). The quantity $\sigma$ is the sought apparent surface charge since

$$
\begin{aligned}
E_{I}\left(\rho, \rho^{\prime}\right) & =\int_{\mathbb{R}^{3}} \mathcal{N}_{\rho}(\mathbf{x}) \rho^{\prime}(\mathbf{x}) d \mathbf{x}+\int_{\mathbb{R}^{3}} \rho^{\prime}(\mathbf{x})(\mathcal{V} \sigma)(\mathbf{x}) d \mathbf{x} \\
& =\int_{\mathbb{R}^{3}} \mathcal{N}_{\rho}(\mathbf{x}) \rho^{\prime}(\mathbf{x}) d \mathbf{x}+\int_{\mathbb{R}^{3}} \rho^{\prime}(\mathbf{x})\left\{u(\mathbf{x})-\mathcal{N}_{\rho}(\mathbf{x})\right\} d \mathbf{x} \\
& =\int_{\mathbb{R}^{3}} \rho^{\prime}(\mathbf{x}) u(\mathbf{x}) d \mathbf{x} .
\end{aligned}
$$

Remark 2 (a) It holds

$$
\sigma=\frac{\partial u_{i}}{\partial \mathbf{n}}-\frac{\partial u_{e}}{\partial \mathbf{n}}=\frac{\epsilon-1}{\epsilon} \frac{\partial u_{i}}{\partial \mathbf{n}}
$$

The first identity is seen by subtracting the Dirichlet-to-Neumann maps (3.4) and inserting the interface condition (2.3) and the definition $\sigma=\mathcal{V}^{-1}\left(u_{i}-\mathcal{N}_{\rho}\right)$ which gives

$$
\mathcal{V}\left\{\frac{\partial u_{i}}{\partial \mathbf{n}}-\frac{\partial u_{e}}{\partial \mathbf{n}}\right\}=\left(\frac{1}{2}+\mathcal{K}\right) u_{i}-\mathcal{N}_{\rho}-\left(\mathcal{K}-\frac{1}{2}\right) u_{e}=u_{i}-\mathcal{N}_{\rho}=\mathcal{V} \sigma \quad \text { on } \Gamma
$$


The second identity follows immediately from the interface condition (2.3).

(b) Since $\mathcal{N}_{\rho}$ is harmonic in $\Omega^{c}$, there holds

$$
\mathcal{V} \frac{\partial \mathcal{N}_{\rho}}{\partial \mathbf{n}}=\left(\mathcal{K}-\frac{1}{2}\right) \mathcal{N}_{\rho} \quad \text { on } \Gamma .
$$

Thus, employing the Calderón identity $\mathcal{V} \mathcal{K}^{\star}=\mathcal{K} \mathcal{V}$, the integral equation (3.3) can be rewritten as a second kind integral equation

$$
\mathcal{A}^{\star} \sigma=\frac{\partial \mathcal{N}_{\rho}}{\partial \mathbf{n}} \text { on } \Gamma
$$

This equation is however in practice more complicated to evaluate since the computation of the electric field $\partial \mathcal{N}_{\rho} / \partial \mathbf{n}$ is expensive.

\section{Local shape derivative}

We shall now assume that the cavity $\Omega$ varies. This happens, for example, if derivatives with respect to the nuclei positions need to be computed. We apply a shape sensitivity analysis to compute the interaction energy's sensitivity with respect to the shape $\Omega$. For a general overview on shape calculus, mainly based on the perturbation of identity (Murat and Simon) or the speed method (Sokolowski and Zolesio), we refer the reader for example to $[6,12,13,15,16]$ and the references therein.

For a smooth perturbation field $\mathbf{V} \in C^{1}\left(\mathbb{R}^{3}\right)$ we define the perturbed domain $\Omega_{\varepsilon}$ according to

$$
\Omega_{\varepsilon}:=\{\mathbf{y}=\mathbf{x}+\varepsilon \mathbf{V}(\mathbf{x}): \mathbf{x} \in \Omega\},
$$

where $\varepsilon>0$ is a sufficiently small parameter. Since $u=u(\Omega)$ depends on the domain $\Omega$, the interaction energy (2.2) depends itself on the domain. Its shape derivative can now be simply expressed in terms of the local shape derivative

$$
\delta E_{I}\left(\rho, \rho^{\prime}\right)[\mathbf{V}]=\int_{\mathbb{R}^{3}} \delta u(\mathbf{x}) \rho^{\prime}(\mathbf{x}) \mathrm{d} \mathbf{x} .
$$

The local shape derivative $\delta u=\delta u[\mathbf{V}]$ is pointwisely defined as

$$
\delta u(\mathbf{x})=\lim _{\varepsilon \rightarrow 0} \frac{u\left(\Omega_{\varepsilon}, \mathbf{x}\right)-u(\Omega, \mathbf{x})}{\varepsilon}, \quad \mathbf{x} \in\left(\Omega \cap \Omega_{\varepsilon}\right) \cup\left(\Omega^{c} \cap \Omega_{\varepsilon}^{c}\right)
$$

and can be computed as the solution of a boundary value problem, see $[9,10]$. 
Theorem 3 Let $\Omega$ be $C^{1, \alpha}$-smooth and $\mathbf{V} \in C^{1}\left(\mathbb{R}^{3}\right)$. Then, the local shape derivative $\delta u=\delta u[\mathbf{V}]$ of (2.3) reads as

$$
\begin{array}{ll}
\Delta \delta u_{i}=0 & \text { in } \Omega, \\
\Delta \delta u_{e}=0 & \text { in } \Omega^{c}, \\
\delta u_{i}=\delta u_{e}-\langle\mathbf{V}, \mathbf{n}\rangle \sigma & \text { on } \Gamma, \\
\frac{\partial \delta u_{i}}{\partial \mathbf{n}}=\epsilon \frac{\partial \delta u_{e}}{\partial \mathbf{n}}-\operatorname{div}_{\Gamma}\left((\epsilon-1)\langle\mathbf{V}, \mathbf{n}\rangle \nabla_{\Gamma} u\right)+\langle\mathbf{V}, \mathbf{n}\rangle \rho & \text { on } \Gamma, \\
\left|\delta u_{e}(\mathbf{x})\right|=\mathcal{O}\left(\|\mathbf{x}\|^{-1}\right) & \text { as }\|\mathbf{x}\| \rightarrow \infty
\end{array}
$$

\section{Proof Setting}

$$
a(\mathbf{x})=\left\{\begin{array}{ll}
1, & \mathbf{x} \in \Omega, \\
\epsilon, & \mathbf{x} \in \Omega^{c},
\end{array} \quad f(\mathbf{x})= \begin{cases}\rho(\mathbf{x}), & \mathbf{x} \in \Omega, \\
0, & \mathbf{x} \in \Omega^{c},\end{cases}\right.
$$

we can rewrite the transmission problem (2.3) according to

$$
\begin{array}{ll}
-\operatorname{div}(a \nabla u)=f & \text { in } \mathbb{R}^{3}, \\
{[u]_{ \pm}=0,\left[a \frac{\partial u}{\partial \mathbf{n}}\right]_{ \pm}=0} & \text { on } \Gamma, \\
|\delta u(\mathbf{x})|=\mathcal{O}\left(\|\mathbf{x}\|^{-1}\right) & \text { as }\|\mathbf{x}\| \rightarrow \infty
\end{array}
$$

where $[\cdot]_{ \pm}$denotes the jump across the interface $\Gamma$. The shape derivative $\delta u$ of this boundary value problem has been computed in $[9,10]$. It is given by

$$
-\operatorname{div}(a \nabla \delta u)=0 \text { in } \mathbb{R}^{3}, \quad|\delta u(\mathbf{x})|=\mathcal{O}\left(\|\mathbf{x}\|^{-1}\right) \text { as }\|\mathbf{x}\| \rightarrow \infty
$$

together with the jump conditions

$$
\left.\begin{array}{l}
{[\delta u]_{ \pm}=-\langle\mathbf{V}, \mathbf{n}\rangle\left[\frac{\partial u}{\partial \mathbf{n}}\right]_{ \pm}} \\
{\left[a \frac{\partial \delta u}{\partial \mathbf{n}}\right]_{ \pm}=\operatorname{div}_{\Gamma}\left(\langle\mathbf{V}, \mathbf{n}\rangle[a]_{ \pm} \nabla_{\Gamma} u\right)+\langle\mathbf{V}, \mathbf{n}\rangle[f]_{ \pm}}
\end{array}\right\} \quad \text { on } \Gamma
$$

In view of (3.5) it holds

$$
[\delta u]_{ \pm}=\delta u_{e}-\delta u_{i}=-\langle\mathbf{V}, \mathbf{n}\rangle\left\{\frac{\partial u_{e}}{\partial \mathbf{n}}-\frac{\partial u_{i}}{\partial \mathbf{n}}\right\}=\langle\mathbf{V}, \mathbf{n}\rangle \sigma
$$

which is the first interface condition in (4.2). Similarly, due to $[a]_{ \pm}=\epsilon-1$ and $[f]_{ \pm}=-\rho$, we conclude the second interface condition

$$
\left[a \frac{\partial \delta u}{\partial \mathbf{n}}\right]_{ \pm}=\epsilon \frac{\partial \delta u_{e}}{\partial \mathbf{n}}-\frac{\partial \delta u_{i}}{\partial \mathbf{n}}=\operatorname{div}_{\Gamma}\left((\epsilon-1)\langle\mathbf{V}, \mathbf{n}\rangle \nabla_{\Gamma} u\right)-\langle\mathbf{V}, \mathbf{n}\rangle \rho
$$

Inserting both expressions into (4.3), we arrive at the transmission problem (4.2). 


\section{Shape gradient}

With (4.1) and (4.2) at hand, we can compute the interaction energy's sensitivity on a given shape variation $\mathbf{V} \in C^{1}\left(\mathbb{R}^{3}\right)$. However, for each $\mathbf{V}$ the boundary value problem (4.2) has to be solved which is quite expensive. We can overcome this obstruction by introducing the so-called adjoint state which leads to the Hadamard representation formula of the interaction energy's shape gradient.

Theorem 4 The Hadamard representation formula of the interaction energy's shape gradient reads as

$$
\delta E_{I}\left(\rho, \rho^{\prime}\right)[\mathbf{V}]=\int_{\Gamma}\langle\mathbf{V}, \mathbf{n}\rangle\left\{(\epsilon-1)\left\langle\nabla_{\Gamma} u_{i}, \nabla_{\Gamma} p_{i}\right\rangle+\rho p_{i}+\sigma \frac{\partial p_{i}}{\partial \mathbf{n}}\right\} \mathrm{d} o
$$

where the adjoint state $p=p(\Omega)$ satisfies the transmission problem

$$
\begin{array}{ll}
-\Delta p_{i}=\rho^{\prime} & \text { in } \Omega, \\
\Delta p_{e}=0 & \text { in } \Omega^{c}, \\
\epsilon p_{i}=p_{e}, \frac{\partial p_{i}}{\partial \mathbf{n}}=\frac{\partial p_{e}}{\partial \mathbf{n}} & \text { on } \Gamma, \\
\left|p_{e}(\mathbf{x})\right|=\mathcal{O}\left(\|\mathbf{x}\|^{-1}\right) & \text { as }\|\mathbf{x}\| \rightarrow \infty .
\end{array}
$$

Proof From (4.1) we find, in view of (5.2), by Green's second idendity

$$
\begin{aligned}
\delta E_{I}\left(\rho, \rho^{\prime}\right)[\mathbf{V}]= & \int_{\mathbb{R}^{3}} \delta u(\mathbf{x}) \rho^{\prime}(\mathbf{x}) \mathrm{d} \mathbf{x} \\
= & -\int_{\Omega \cup \Omega^{c}} \delta u(\mathbf{x}) \Delta p(\mathbf{x}) \mathrm{d} \mathbf{x} \\
= & -\int_{\Omega \cup \Omega^{c}} \Delta \delta u(\mathbf{x}) p(\mathbf{x}) \mathrm{d} \mathbf{x}+\int_{\Gamma} \frac{\partial \delta u_{i}}{\partial \mathbf{n}} p_{i} \mathrm{~d} o-\int_{\Gamma} \delta u_{i} \frac{\partial p_{i}}{\partial \mathbf{n}} \mathrm{d} o \\
& -\int_{\Gamma} \frac{\partial \delta u_{e}}{\partial \mathbf{n}} p_{e} \mathrm{~d} o+\int_{\Gamma} \delta u_{e} \frac{\partial p_{e}}{\partial \mathbf{n}} \mathrm{d} o .
\end{aligned}
$$

The domain integral vanishes due to $\Delta \delta u_{i}=\Delta \delta u_{e}=0$. By inserting the jump conditions of $p$ and afterwords that of $\delta u$, we conclude

$$
\begin{aligned}
\delta E_{I}\left(\rho, \rho^{\prime}\right)[\mathbf{V}] & =\int_{\Gamma}\left\{\frac{\partial \delta u_{i}}{\partial \mathbf{n}}-\epsilon \frac{\partial \delta u_{e}}{\partial \mathbf{n}}\right\} p_{i} \mathrm{~d} o+\int_{\Gamma}\left\{\delta u_{e}-\delta u_{i}\right\} \frac{\partial p_{i}}{\partial \mathbf{n}} \mathrm{d} o \\
& =\int_{\Gamma}\left\{\langle\mathbf{V}, \mathbf{n}\rangle \rho-\operatorname{div}_{\Gamma}\left((\epsilon-1)\langle\mathbf{V}, \mathbf{n}\rangle \nabla_{\Gamma} u_{i}\right)\right\} p_{i} \mathrm{~d} o+\int_{\Gamma}\langle\mathbf{V}, \mathbf{n}\rangle \sigma \frac{\partial p_{i}}{\partial \mathbf{n}} \mathrm{d} o .
\end{aligned}
$$

Integrating the first integral by parts yields finally the desired Hadamard representation fomula (5.1). 
Remark 5 The shape gradient (5.1) involves the term $\left.\left(\rho p_{i}\right)\right|_{\Gamma}$ which stems from the jump of the charge $\rho$ across the interface. Since, however, the charge is in practice continuous across the interface, it might make sense to discard this term.

\section{Boundary integral equation formulations}

According to Theorem 4, in addition to the apparent surface charge $\sigma$, the shape gradient involves the functions $u_{i}, p_{i}$, and $\partial p_{i} / \partial \mathbf{n}$ on $\Gamma$. We shall therefore provide appropriate boundary integral equations for their computation.

In order to compute the function $u_{i}$ we can use the Dirichlet-to-Neumann maps (3.4). Substracting $\epsilon$ times the exterior Dirichlet-to-Neumann map from the interior one yields, in view of the jump condition (2.3), the boundary integral equation

$$
\mathcal{A} u_{i}=\frac{1}{\epsilon-1} \mathcal{N}_{\rho} \text { on } \Gamma
$$

with $\mathcal{A}$ being defined in (3.3).

The function $p_{i}$ can be computed in complete analogy by the use of (3.4). Observing the jump condition (5.2), we substract the exterior from the interior Dirichlet-toNeumann map and arrive at

$$
\mathcal{A} p_{i}=\frac{1}{\epsilon-1} \mathcal{N}_{\rho^{\prime}} \text { on } \Gamma \text {. }
$$

Likewise, subtracting the exterior from $\epsilon$ times the interior Dirichlet-to-Neumann map (3.4) and inserting the jump condition (5.2) and relation (6.2) leads to the boundary integral equation

$$
\frac{\epsilon-1}{\epsilon} \mathcal{V} \frac{\partial p_{i}}{\partial \mathbf{n}}=\frac{1}{\epsilon-1} \mathcal{A}^{-1} \mathcal{N}_{\rho^{\prime}}-\mathcal{N}_{\rho^{\prime}}=p_{i}-\mathcal{N}_{\rho^{\prime}} \quad \text { on } \Gamma .
$$

Hence, both boundary integral equations (6.2) and (6.3), related with the adjoint state, correspond to the equations for the primal state, cf. (3.3), (3.5), and (6.1). In particular, for a numerical scheme, we only need to assemble system matrices associated with the boundary integral operators $\mathcal{A}$ and $\mathcal{V}$.

Remark 6 It is stringent to use continuous boundary elements for the discretization of the boundary integral equations (6.1) and (6.2) in order to be able to compute their solutions' surface gradients. However, the integral equation (6.3) for the Neumann data of the adjoint state and the integral equation (3.3) for the apparent surface charge are the same except for scaling. Therefore, only a solver for the boundary integral equation (3.3) (by solving (6.1) as an intermediate step) needs to be implemented in order to compute both, the interaction energy and the shape gradient (5.1). 


\section{References}

1. E. Cancés, B. Mennucci, New applications of integral equations methods for solvation continuum models: ionic solutions and liquid crystals. J. Math. Chem. 23, 309-326 (1998)

2. E. Cancés, B. Mennucci, Analytical derivatives for geometry optimization in solvation continuum models. I. Theory. J. Chem. Phys. 109, 249-259 (1998)

3. E. Cancés, B. Mennucci, Analytical derivatives for geometry optimization in solvation continuum models. II. Numerical applications. J. Chem. Phys. 109, 260-266 (1998)

4. E. Cancés, B. Mennucci, J. Tomasi, A new integral equation formalism for the polarizable continuum model: Theoretical background and applications to isotropic and anisotropic dielectrics. J. Chem. Phys. 107, 3032-3041 (1997)

5. M. Connolly, Analytical molecular surface calculation. J. Appl. Cryst. 16, 548-558 (1983)

6. M. Delfour, J.-P. Zolesio, Shapes and Geometries (SIAM, Philadelphia, 2001)

7. E. DiBenedetto, C.M. Elliot, A. Friedman, The free boundary of a flow in a porous body heated from its boundary. Nonlinear Anal. 10, 879-900 (1986)

8. L. Frediani, R. Cammi, S. Corni, J. Tomasi, A polarizable continuum model for molecules at diffuse interfaces. J. Chem. Phys. 120, 3893-3907 (2004)

9. F. Hettlich, W. Rundell, The determination of a discontinuity in a conductivity from a single boundary measurement. Inverse Probl 14, 67-82 (1998)

10. F. Hettlich, W. Rundell, Identification of a discontinuous source in the heat equation. Inverse Probl 17, 1465-1482 (2001)

11. S. Miertuš, E. Scrocco, J. Tomasi, Electrostatic interaction of a solute with a continuum. A direct utilization of ab initio molecular potentials for the prevision of solvent effects. Chem. Phys. 55, 117-129 (1981)

12. F. Murat, J. Simon, Étude de problèmes d'optimal design, in Optimization Techniques, Modeling and Optimization in the Service of Man, Lecture Notes in Computer Science, vol 41, ed. by J. Céa (Springer, Berlin, 1976), pp. 54-62

13. O. Pironneau, Optimal Shape Design for Elliptic Systems (Springer, New York, 1983)

14. S. Sauter, C. Schwab, Boundary Element Methods (Springer, Berlin, 2011)

15. J. Simon, Differentiation with respect to the domain in boundary value problems. Numer. Funct. Anal. Optim. 2, 649-687 (1980)

16. J. Sokolowski, J.-P Zolesio, Introduction to Shape Optimization (Springer, Berlin, 1992)

17. J. Tomasi, B. Mennucci, R. Cammi, Quantum mechanical continuum solvation models. Chem. Rev. 105, 2999-3094 (2005) 